DOI: 10.1007/s00350-013-3462-9

\section{Kein Nachbesserungsrecht bei Unbrauchbarkeit des Zahnersatzes}

BGB §§249, 253, 276, 280, 611, 823

1. Soweit der Zahnersatz unbrauchbar ist, besteht kein Honoraranspruch des Zahnarztes.

2. Eine Unbrauchbarkeit liegt immer vor, soweit eine Neuanfertigung erforderlich ist.

3. Wenn eine Unbrauchbarkeit des Zahnersatzes vorliegt, kommt ein Nachbesserungsrecht des Zahnarztes nicht mehr in Betracht. (Leitsätze des Bearbeiters)

KG, Beschl. v. 14.1.2013 - 20 W 83/12 (LG Berlin)

Problemstellung: Bei fehlerhaftem Zahnersatz macht der Zahnarzt häufig ein Nachbesserungsrecht geltend. Der Patient möchte aber oft die Behandlung bei diesem Zahnarzt gar nicht fortsetzen. In der Folge kommt es häufig zum Streit über das Honorar. Nach der bisher herrschenden Meinung steht dem Zahnarzt trotz Annahme eines Dienstvertrags kraft Rechtsfortbildung ein Nachbesserungsrecht zu. Fraglich ist, ob das Nachbesserungsrecht auch erlöschen kann oder verwirkt werden kann. Das KG vertritt in ständiger Rechtsprechung die Auffassung, dass kein Nachbesserungsrecht mehr besteht, wenn der Zahnersatz unbrauchbar ist. In dem hier entschiedenen Fall musste das KG entscheiden, was passiert, wenn eine Teilunbrauchbarkeit des Zahnersatzes vorliegt.

Zum Sachverhalt: Im Jahre 2010 suchte der Bekl. den Zahnarzt Dr. E. auf, weil er sich das Zahngebiss sanieren lassen wollte. Die Behandlung wurde vom 16.8.2010 bis zum 18.10.2010 durchgeführt. Am 12.10.2010 wurden Brücken und Kronen sowie die Keramikinlays im Unterkiefer befestigt. Wegen erheblicher Schmerzen und Beschwerden des Bekl. kam es zu einer erneuten Untersuchung am 18. 10.2010. Der Bekl. monierte die Arbeiten als mangelhaft. Dies wies der Zahnarzt jedoch zurück. Daraufhin verließ der Bekl. die Zahnarztpraxis von Dr. E.

Danach suchte der Bekl. eine andere Zahnarztpraxis auf und ließ sich dort eine zweite Meinung geben. Der zweite Zahnarzt meinte, die Brücken im Oberkiefer seien derart stark eingeschliffen, dass eine Neuanfertigung erforderlich sei. Der Bekl. suchte dann einen Anwalt auf, der ein selbständiges Beweisverfahren über den Zustand der eingesetzten Zahnprothetik beim LG einleitete. Die Sachverständige Frau Prof. Dr. N. kam zum Ergebnis, dass die eingesetzte Zahnprothetik zahnmedizinische Mängel aufweise, insbesondere, dass die Brücken im Oberkiefer sowie die Krone auf Zahn 46 zu erneuern seien. Trotz Kenntnis dieses Gutachtens klagte die Inkassostelle, an die die Honorarforderung abgetreten worden war, die Forderung ein. Der Bekl. stellte einen Antrag auf Prozesskostenhilfe für seine Verteidigung unter Beifügung des Gutachtens aus dem selbständigen Beweisverfahren.

Das LG wies den Antrag des Bekl. auf Prozesskostenhilfe für seine Verteidigung gegen die Honorarklage ab. Bei einem Zahnarztvertrag sei das Honorar grundsätzlich geschuldet, es sei denn der Zahnersatz sei völlig unbrauchbar. Aus dem Gutachten ließen sich dafür keine Anhaltspunkte entnehmen. Selbst wenn eine Neuanfertigung erfolgen müsse, könne es ausnahmsweise ein Nachbesserungsrecht des Zahnarztes geben. Dies gelte erst dann nicht mehr, wenn die Fortsetzung der Behandlung für den Patienten unzumutbar sei. Hierzu habe der Bekl. nichts vorgetragen.

Dagegen richtete sich die Beschwerde des Bekl.

Aus den Gründen: Die Beschwerde musste teilweise Erfolg haben. Die Rechtsverteidigung des Bekl. hat hin-

Eingesandt und bearbeitet von Rechtsanwalt Dominik Kellner, Fachanwalt für Medizinrecht, Kanzlei für Patientenrecht, Goethestraße 58, 10625 Berlin, Deutschland reichende Aussicht auf Erfolg, soweit er sich gegen die auf Herstellung der Oberkieferbrücke gestützte, an die Kl. abgetretene Honorarforderung des Zahnarztes wendet. Die Sachverständige Prof. Dr. N. hat im selbständigen Beweisverfahren $35 \mathrm{OH}$ 5/11 durch Gutachten v. 31. 10.2011 festgestellt, dass die Oberkieferbrücken nach einer angemessenen Wartezeit nach erneuter Wurzelkanalbehandlung zu erneuern sind, und dass dies auch für die Krone auf Zahn 46 gilt. Mithin kann unter weiterer Berücksichtigung der Ausführungen der Sachverständigen zu den Fehlerursachen nicht ausgeschlossen werden, dass die Oberkieferbrücken behandlungsfehlerhaft hergestellt wurden. Hierüber hat das LG ggf. Beweis zu erheben.

Danach wäre der Honoraranspruch unbegründet, soweit er auf Bezahlung einer für den Bekl. infolge eines Behandlungsfehlers unbrauchbare[n] Leistung, nämlich die Oberkieferbrücke gerichtet ist. Das darauf gestützte Teilhonorar ist aus dem Gesamtanspruch herauszurechnen (vgl. auch Senat, Beschl. v. 1.7.2010 - 20 W 23/10 -).

Der teilweisen Bewilligung von Prozesskostenhilfe steht nicht entgegen, dass die Rechtsverteidigung des Bekl. nur gegenüber einem Teilanspruch der Kl. erfolgreich erscheint, und dass sich dieser Teil zur Zeit betragsmäßig nicht hinreichend darstellt. Zwar darf Prozesskostenhilfe nicht für unselbständige Verfahrensabschnitte bewilligt werden, wie etwa eine von mehreren Einwendungen (Geimer, in: Zöller, ZPO, 29. Aufl. 2012, \$114, Rdnr. 20). Dies kann jedoch nicht gelten, soweit sich die Verteidigung, wie hier, gegen sachlich und betragsmäßig abgrenzbare Teile eines Gesamtanspruches richtet, für die auch dem Anspruchssteller unter den geforderten Voraussetzungen Prozesskostenhilfe zu bewilligen wäre.

\section{[...]}

Der Senat hat von der Erhebung einer Gebühr für dieses Verfahren abgesehen, weil die Beschwerde mit Blick auf die Gesamt-Honorarrechnung überwiegend Erfolg hat (KV Nr. 1811).

\section{Befunderhebungsversäumnisse bei Leitsymptomen für spinale Infektion und grobe Behandlungsfehler - Beweislastumkehr; Verjährungsbeginn}

BGB §§ 195, 199, 249, 253, 276, 278, 280, 611, 823, 831, 847 a. F., 852 a. F.; ZPO §§286, 287

1. Gelangt ein Arzt nach einer abgeklungenen Staphylokokkeninfektion des Patienten zu der nicht vorwerfbar falschen Diagnose ,Kyphose und Spondylose", nimmt er jedoch die durch Bildgebung gesicherte Progredienz einer Wirbelkörperdestruktion nebst Rückenschmerzen sowie das auffällige Ergebnis einer Lumbalpunktion nicht zum Anlass, dem Verdacht einer Spondylodiszitis nachzugehen, hat dieses Versäumnis eine Beweislastumkehr zur Folge.

2. Treten bei einem in multipler Weise vorgeschädigten Patient zusätzliche erhebliche Ausfallerscheinungen mit stärksten Schmerzen auf, was das Erfordernis einer operativen Intervention nahe legt, handelt es sich bei der um Stunden verzögerten Verlegung in eine hierfür geeignete Klinik um einen groben Behandlungsfehler.

Bearbeitet von VorsRiBGH a.D. Dr. iur. Erich Steffen, Kriegsstraße 258, 76135 Karlsruhe, Deutschland 